\title{
Topological Measure Locating the Effective Crossover between Segregation and Integration in a Modular Network
}

\author{
A. Adjari Rad, ${ }^{1}$ I. Sendiña-Nadal, ${ }^{2,3}$ D. Papo, ${ }^{3}$ M. Zanin, ${ }^{3}$ J. M. Buldú,,${ }^{2,3}$ F. del Pozo, ${ }^{3}$ and S. Boccaletti ${ }^{3}$ \\ ${ }^{1}$ École Polytechnique Fédéral de Lausanne, Laboratory of Nonlinear Systems, School of Computer and Communication Science, \\ 1015 Lausanne, Switzerland \\ ${ }^{2}$ Complex Systems Group, Universidad Rey Juan Carlos, 28933 Móstoles, Madrid, Spain \\ ${ }^{3}$ Center for Biomedical Technology, Universidad Politécnica de Madrid, \\ Campus de Montegancedo, 28223 Pozuelo de Alarcón, Madrid, Spain
}

(Received 1 December 2011; published 29 May 2012)

\begin{abstract}
We introduce an easily computable topological measure which locates the effective crossover between segregation and integration in a modular network. Segregation corresponds to the degree of network modularity, while integration is expressed in terms of the algebraic connectivity of an associated hypergraph. The rigorous treatment of the simplified case of cliques of equal size that are gradually rewired until they become completely merged, allows us to show that this topological crossover can be made to coincide with a dynamical crossover from cluster to global synchronization of a system of coupled phase oscillators. The dynamical crossover is signaled by a peak in the product of the measures of intracluster and global synchronization, which we propose as a dynamical measure of complexity. This quantity is much easier to compute than the entropy (of the average frequencies of the oscillators), and displays a behavior which closely mimics that of the dynamical complexity index based on the latter. The proposed topological measure simultaneously provides information on the dynamical behavior, sheds light on the interplay between modularity and total integration, and shows how this affects the capability of the network to perform both local and distributed dynamical tasks.
\end{abstract}

DOI: 10.1103/PhysRevLett.108.228701

PACS numbers: 89.75.Hc, 05.45.Xt, 89.70.Eg, 89.75.Fb

Many physical and biological systems (such as electronic devices, communications networks, and the human brain) face similar constraints as they interact with complex environments, and organize their structure and function along similar principles of resource allocation [1]. On the one hand, the need for fast and reliable responses to changes in the environment naturally favors the emergence of segregated modules of specialized computation (e.g., sensory systems in the brain). On the other hand, interactions among modules become essential when an information processing whose complexity exceeds the capacity of the single modules is required. For instance, perceptual systems in the brain need to bind information from different brain areas to produce a single coherent percept [2]. Therefore, segregation into specialized modules and integration into global coherent activity present an inherent trade-off, and an appropriate balance between these two tendencies has been shown to be necessary for efficient functioning, particularly in neural systems [3]. In fact, an exceedingly segregated or integrated functioning of the brain has been associated with various pathological conditions, e.g., autism or schizophrenia [4-6], and epilepsy [7], respectively.

One straightforward way to study such a balance in complex systems is to represent them as dynamical networks, endowing them with well-studied topological and dynamical properties (see [8,9] for a review). For instance, Zhao et al. [10] characterized systems of coupled phase oscillators in terms of a complexity index based on the entropy of the distribution of pairwise synchronization. Heterogeneous and modular networks were shown to be characterized by high complexity, for intermediate levels of modularity, in a regime marked by the formation of dynamical clusters and the coordination between them.

In this Letter, we provide an easily computable topological measure for quantifying the balance between segregation and integration in a network. We propose that segregation can be understood in terms of a community structure (i.e., clusters of vertices densely connected to each other while less connected to vertices outside the community [11]), while integration can conveniently be expressed in terms of algebraic connectivity [12] of the hypergraph associated with the network. After introducing our measure for a generic modular graph, we focus on the simplified case in which the network communities are of equal size and have the same number of inner and outer connections, and show analytically that there is a structure that maximizes the product of segregation and coordination measures. We then demonstrate that the dynamics emerging from such a specific configuration is associated with the coincidence of the two thresholds for cluster and complete synchronization in a network of interacting phase oscillators.

We start by considering a generic undirected, unweighted graph $G$ composed of $N$ nodes and $L$ links, partitioned into $C$ communities, and characterized by an 


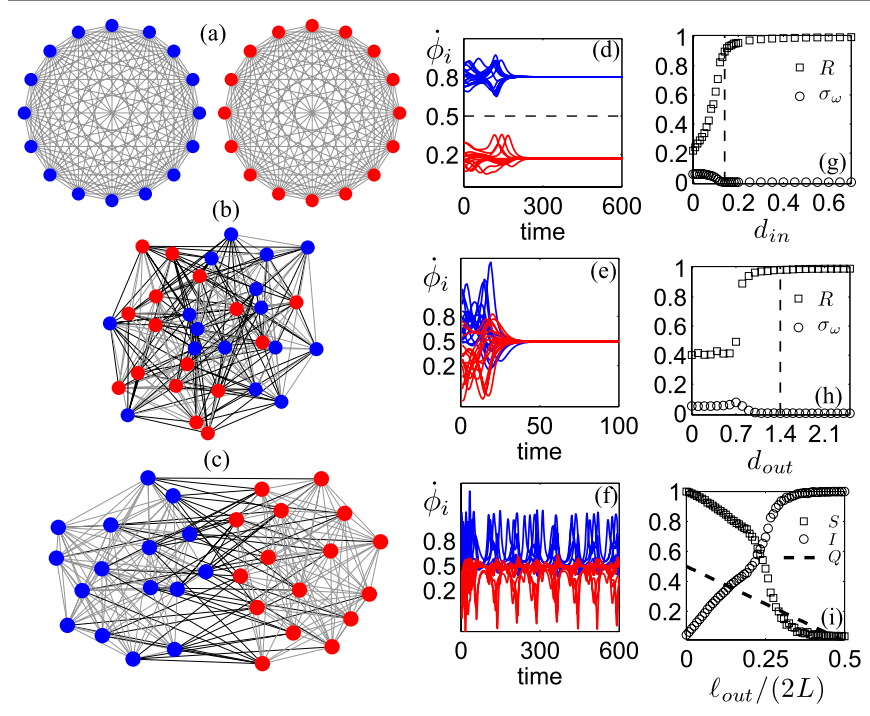

FIG. 1 (color online). (a)-(c) Graph representations of (a) a network consisting of two cliques of size $N_{C}=16$, and (b-c) the resulting networks after rewiring. (b) $j^{\max }=60\left(\ell_{\text {out }} / 2 L=0.5\right.$ and $Q=0)$, and (c) $j=30\left(\ell_{\text {out }} / 2 L=0.25\right.$ and $\left.Q=0.25\right)$. Intracommunity (intercommunity) links are light gray (black colored). Blue (red) nodes are phase oscillators whose natural frequency is randomly distributed around $0.8(0.2)$ within a range \pm 0.1 . (d)-(f) Time evolution of the instantaneous frequencies of the $N=32$ oscillators after solving Eq. (4) with the networks of the left panels and $d_{\text {in }}=d_{\text {in }}^{*}=0.14$. (d) $d_{\text {out }}=0$, (e) $d_{\text {out }}=d_{\text {out }}^{*}=1.10$, and (f) $d_{\text {out }}=1.40$. Dashed line in (d) marks the mean frequency between the two clusters. (g)-(h) $R$ (squares) and $\sigma_{\omega}$ (circles) (see text for definition) for: (g) the two clique network of (a) as a function of $d_{\text {in }}$, (h) the 0-modularity network of (b) as a function of $d_{\text {out }}$ and for $d_{\text {in }}=0.14$. The vertical dashed line in (g) marks the critical coupling for cluster synchronization $\left(d_{\text {in }}^{*}=0.14\right)$, while in (h) it corresponds to $d_{\text {out }}=1.4$, well above the onset of global synchronization $\left(d_{\text {out }}^{*}=1.10\right.$ ). Panel (i) reports the $S$ and $I$ indices (see text for definitions) vs the ratio $\ell_{\text {out }} /(2 L)$, after solving Eq. (4) with $d_{\text {in }}=0.14$ and $d_{\text {out }}=1.40$. Oblique dashed line is the corresponding modularity index $Q$.

associated $N \times N$ adjacency matrix $A$. Moreover, for each community $r(r=1, \ldots, C)$, we consider the number of links connecting pairs of members of that community $\left(\ell_{\mathrm{in}}^{r}\right)$, and the number of intercommunity links $\left(\ell_{\text {out }}^{r}\right)$, i.e., the number of links connecting a member of that community with a member of another community. Based on the mentioned notation, we have $L=\left(\sum_{r=1}^{C} \ell_{\text {in }}^{r}\right)+\frac{1}{2}\left(\sum_{r=1}^{C} \ell_{\text {out }}^{r}\right)$. Using the previous definitions, the standard modularity measure defined in [13] can be written as $Q=$ $\sum_{r=1}^{C}\left\{\ell_{\text {in }}^{r} / L-\left[\left(2 \ell_{\text {in }}^{r}+\ell_{\text {out }}^{r}\right) / 2 L\right]^{2}\right\}$.

Let us now define the hypergraph $G^{*}$ associated to $G$, as the weighted directed $C$ clique in which each node corresponds to a community of $\mathcal{G}$, and the connection incident to node $r$ from node $s$ is weighted by $\frac{\ell_{\text {out }}^{r s}}{\ell_{\text {in }}^{r}}, \ell_{\text {out }}^{r s}$ being the number of links of $G$ that connects members of the community $r$ with members of the community $s$, and $\ell_{\text {in }}^{r}$ the number of inner links in the source community. The corresponding $C \times C$ Laplacian matrix $\mathcal{L}^{*}=\left\{\mathcal{L}_{r s}^{*}\right\}$ is asymmetric, but can be written as the product $\mathcal{L}^{*}=\mathcal{B C}$, where $\mathcal{C}$ is a symmetric zero-row sum matrix with off-diagonal elements $\mathcal{C}_{r s}=-\ell_{\text {out }}^{r s}$ and diagonal elements $\mathcal{C}_{r r}=\ell_{\text {out }}^{r}=$ $\sum_{s \neq r} \ell_{\text {out }}^{r s}$, and $\mathcal{B}=\operatorname{diag}\left\{1 / \ell_{\text {in }}^{1}, \ldots, 1 / \ell_{\text {in }}^{C}\right\}$ :

$\mathcal{L}^{*}=\left(\begin{array}{cccc}\frac{1}{\ell_{\text {in }}^{1}} & 0 & \cdots & 0 \\ 0 & \frac{1}{\ell_{\text {in }}^{2}} & \cdots & 0 \\ \vdots & \vdots & \ddots & \vdots \\ 0 & 0 & \cdots & \frac{1}{\ell_{\text {in }}^{C}}\end{array}\right)\left(\begin{array}{cccc}\ell_{\mathrm{out}}^{1} & -\ell_{\mathrm{out}}^{12} & \cdots & -\ell_{\mathrm{out}}^{1 C} \\ -\ell_{\mathrm{out}}^{21} & \ell_{\mathrm{out}}^{2} & \cdots & -\ell_{\mathrm{out}}^{2 C} \\ \vdots & \vdots & \ddots & \vdots \\ -\ell_{\mathrm{out}}^{C 1} & -\ell_{\mathrm{out}}^{C 2} & \cdots & \ell_{\mathrm{out}}^{C}\end{array}\right)$.

The spectrum of $\mathcal{L}^{*}$ is real with non-negative values and because $\mathcal{L}^{*}$ is zero-row sum, the smallest eigenvalue $\lambda_{1}^{*}$ is zero, while $\lambda_{2}^{*}>0$. The measure that we propose for the balance between integration and segregation is defined as follows:

$$
\xi=Q \lambda_{2}^{*}
$$

Indeed, while $Q$ is an inherent evaluation of the segregation factor of a graph, $\lambda_{2}^{*}>0$ quantifies the connectiveness of the hypergraph, and therefore measures the extent to which different communities are bounded and interact. It should be noted that both $Q$ and $\lambda_{2}^{*}$ are properly normalized in such a way that-even if the network links were associated to cohesive forces - the two quantities will be adimensional. The maximum of $\xi$ corresponds to a topology in which integration and segregation have the same weight.

Let us then consider the case in which the $C$ communities are cliques having equal size $N_{c}=N / C$, and the number of intracommunity links, $\ell_{\mathrm{in}}^{r}$, as well as the number of intercommunity links, $\ell_{\text {out }}^{r}$, are the same for all communities. We then have $\ell_{\mathrm{in}}^{r}=\ell_{\mathrm{in}} / C$, and $\ell_{\mathrm{out}}^{r}=\ell_{\mathrm{out}} / C$, such that $L=C\left(\ell_{\text {in }}^{r}+\frac{1}{2} \ell_{\text {out }}^{r}\right)=\ell_{\text {in }}+\frac{1}{2} \ell_{\text {out }}$. Under these assumptions, the modularity can be reduced to the following expression:

$$
Q=C\left[\frac{1}{L} \frac{\ell_{\text {in }}}{C}-\left(\frac{2 L}{C} \frac{1}{2 L}\right)^{2}\right]=1-\frac{1}{2 L} \ell_{\text {out }}-\frac{1}{C} .
$$

On the other hand, we have that $\mathcal{B}=\frac{C}{\ell_{\text {in }}} I$ and, from matrix identity,

$$
\lambda_{2}^{*}=\frac{\ell_{\mathrm{out}}}{\ell_{\mathrm{in}}} .
$$

Let us now consider a particular protocol by means of which $l_{\text {out }}$ is varied from 0 to $2 L(C-1) / C$, the value at which the modularity $Q$ is zero. We start from a fully segregated configuration (in which $\ell_{\text {out }}=0$ and $\ell_{\text {in }}=L$ ), and operate successive rewiring processes, in each of which an intracommunity link from each community is deleted, and $C$ intercommunity links are formed by connecting those pairs of nodes (each one in different 
communities) having lost their intralink. In this way, at the $j$ th rewiring, we have $\ell_{\text {in }}=L-C j$, and $\ell_{\text {out }}=C 2 j$. The maximum number of rewiring steps until modularity fades out is, therefore, $N_{c}\left(N_{c}-1\right)(C-1) /(2 C)$. It follows from combining Eqs. (1) and (2) that $\xi=Q \lambda_{2}^{*}=$ $\left(1-\frac{1}{C}-\frac{1}{2 L} \ell_{\text {out }}\right) \frac{\ell_{\text {out }}}{L-\frac{1}{2} \ell_{\text {out }}}$. Accordingly, the partial derivative of $\xi$ with respect to $\ell_{\text {out }}$ is $\frac{\partial \xi}{\partial \ell_{\text {out }}}=1-\frac{1}{C}-\frac{\ell_{\text {out }}}{L}+\frac{1}{4}\left(\frac{\ell_{\text {out }}}{L}\right)^{2}$, which vanishes at

$$
\ell_{\mathrm{out}}^{\max }=2 L\left(1-\frac{1}{\sqrt{C}}\right) ;
$$

i.e., there exists a value of intercommunity links at which $\xi$ reaches its maximum value (the second derivative of $\xi\left(\ell_{\text {out }}\right)$ is indeed negative).

We now show that the maximization of $\xi$ has a dynamical counterpart for the analytically treated example. To this purpose, we consider a modular network of $N$ nodes initially arranged in two cliques $c_{1}$ and $c_{2}$ of equal size $N_{c}=\frac{N}{2}$ [Fig. 1(a)], with $\ell_{\text {out }}^{c_{1}}=\ell_{\text {out }}^{c_{2}}=0$ and $\ell_{\text {in }}^{c_{1}}=\ell_{\text {in }}^{c_{2}}=$ $N_{c}\left(N_{c}-1\right) / 2$ such that the total number of links is $L=$ $N_{c}\left(N_{c}-1\right)$. The units of the network are taken to be phase oscillators evolving according to the Kuramoto model [14]

$$
\dot{\phi}_{i}=\omega_{i}+\frac{1}{N_{c}-1} \sum_{j=1}^{N} d_{i j} a_{i j} \sin \left(\phi_{j}-\phi_{i}\right)
$$

where $\dot{\phi}_{i}$ is the angular velocity of the $i$ th oscillator, $\omega_{i}$ its natural frequency, $d_{i j}$ is the coupling strength between oscillators $i$ and $j$ (in the following $d_{i j}=d_{\text {in }}$ if the nodes $i$ and $j$ are members of the same community, and $d_{i j}=d_{\text {out }}$ otherwise), and $a_{i j}=1$ if $i$ and $j$ are connected and 0 otherwise. Oscillators $i=1, \ldots, N_{c}\left(i=N_{c}+1, \ldots, N\right)$, initially assigned to clique $c_{1}\left(c_{2}\right)$ and colored in blue (red) in Figs. 1(a)-1(c), have $\omega_{i}$ uniformly distributed around $\omega_{c_{1}}=0.8\left(\omega_{c_{2}}=0.2\right)$ within a range of \pm 0.1 .

To explore the extent of validity of Eq. (3), we start from such a completely segregated configuration, and gradually increase the ratio $\ell_{\text {out }} / 2 L$ by (i) randomly selecting a link in each module, (ii) deleting it, and (iii) forming two new intercommunity links by pairwise connection of the ends of the deleted links. The rewiring process is then repeated up to the point in which the modularity index $Q$ cancels, and both modules become statistically indistinguishable [Fig. 1(b)]. According to Eq. (3), the balance for a $C=2$ module network is found at the ratio $\ell_{\text {out }} / 2 L=1-\sqrt{1 / 2}$.

Furthermore, the tuning of the coupling strengths $d_{\text {in }}$ and $d_{\text {out }}$ allows one to control the degree of intracommunity (cluster) and intercommunity (complete) synchronization in each given configuration. Namely, we compute the order parameter $R=\left\langle\left\|1 / N \sum_{j=1}^{N} e^{i \phi_{j}}\right\|\right\rangle_{T}$ [squares in Figs. 1(g) and $1(\mathrm{~h})]$ and the frequency standard deviation $\sigma_{\omega}$ [circles in Figs. 1(g) and 1(h)], averaged over a proper time window $T$, to account for the phase and frequency synchronization (in our simulations $T=200$ t.u.). In Fig. $1(\mathrm{~g}), d_{\text {in }}$ is increased up to $d_{\text {in }}^{*}=0.14$, which constitutes the threshold for cluster synchronization in the case of the network structure of Fig. 1(a). Figure 1(d) shows that the instantaneous frequencies $\dot{\phi}_{i}$ of all oscillators are locked to their respective cluster frequencies, 0.2 (in red) and 0.8 (in blue). On the other hand, when the modularity index vanishes [as in the network of Fig. 1(b)], we set $d_{\text {in }}$ to $d_{\text {in }}^{*}$ and vary $d_{\text {out }}$ to find the threshold for global synchronization at $d_{\text {out }}^{*}=1.10$ [Fig. 1(h)]. As shown in Fig. 1(e), for $d_{\text {out }}=1.40$ (sufficiently above the transition), the network is performing a collective oscillation at the mean frequency of the two clusters (0.5).

This way, functional segregated and integrated states are guaranteed by choosing $d_{\text {in }}$ and $d_{\text {out }}$ above the threshold couplings for cluster synchronization and global synchronization. Depending on the fraction of intercommunity links, the competition between the dynamics of the individual clusters and the whole network will give rise to a certain degree of functional segregation and integration. Figure 1(f) shows the instantaneous frequencies of the oscillators coupled according to the scheme of Fig. 1(c) for $\ell_{\text {out }} / 2 L=0.25$. One can easily see that the behavior is far from being totally integrated or segregated, but, instead, the instantaneous frequencies of the oscillators undergo high amplitude oscillations around their natural values.

To quantify the degree of dynamical segregation $S$ and dynamical integration $I$, we calculate the ensemble averages $S=\left\langle S_{i}\right\rangle$ and $I=\left\langle I_{i}\right\rangle . S_{i}$ and $I_{i}$ are the dynamical segregation and integration measures for the individual $i$ oscillators, and are defined as $S_{i}=\left\langle\left|\dot{\phi}_{i}-\frac{\omega_{c_{1}}+\omega_{c_{2}}}{2}\right|\right\rangle_{T}$ (the time-averaged absolute distance between the oscillator's instantaneous frequency and the mean frequency of the two clusters), and $I_{i}=\left\langle\left|\dot{\phi}_{i}-\delta\left(s_{i}, c_{1}\right) \omega_{c_{1}}-\delta\left(s_{i}, c_{2}\right) \omega_{c_{2}}\right|\right\rangle_{T}$, where $s_{i}$ is the community of which the oscillator $i$ is a member, and $\delta\left(s_{i}, s_{j}\right)$ the Kronecker delta function. The dependence of these quantities on the mixing ratio $\ell_{\text {out }} / 2 L$ is then normalized, and reported in Fig. 1(i) for $d_{\text {in }}=d_{\text {in }}^{*}$ and $d_{\text {out }}=1.40>d_{\text {out }}^{*}$, showing a monotonically decreasing behavior of $S$ (squares) as the modularity $Q$ (dashed line) vanishes, while a monotonically increasing trend of $I$ (circles) up to saturation when the whole network is fully synchronized.

Consequently, the dynamical segregation or integration trade-off, measured as the product of $S$ and $I$, gives information about the existence of a level of topological mixing for which functional clustering balances global synchronization, as shown in Fig. 2(a) (triangles). An alternative way to measure the combination of dynamical segregation and integration is by means of the complexity index $E$, introduced in Ref. [10] in the context of oscillatory networks. Here, $E$ is calculated using the Shannon entropy of the distribution $P(\omega)$ of the average frequencies of all oscillators as $E=\left(-\sum_{l=1}^{m} P_{l} \ln P_{l}\right) / \ln m$, where $m$ is the number of bins in the histogram of $P(\omega)$. By definition, $E$ 

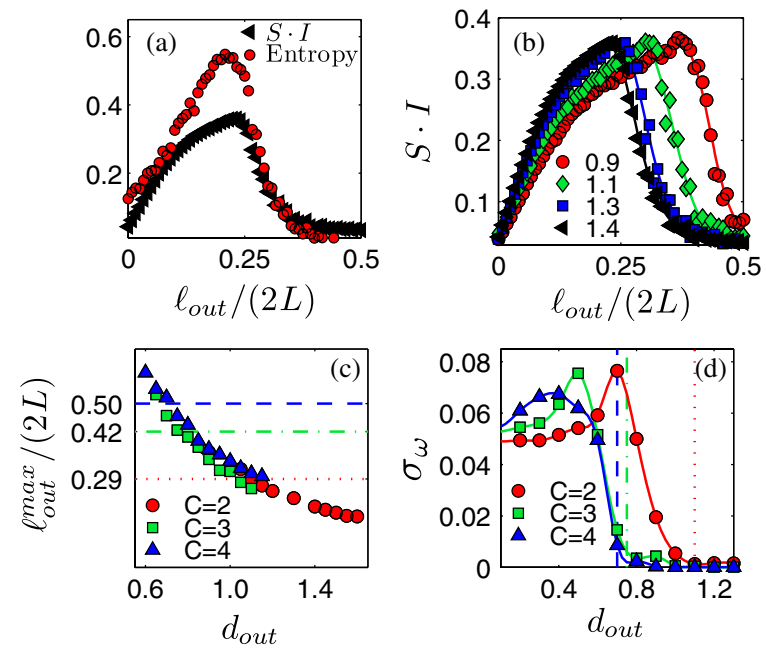

FIG. 2 (color online). (a) Complexity index $E$ (circles) and $S I$ index (triangles) (see text for definitions) corresponding to the data reported in Fig. 1(i). (b) $S I$ curves as a function of the mixing ratio $\ell_{\text {out }} /(2 L)$ for $C=2$ and for increasing values of $d_{\text {out }}$ (in the legend). (c) Maxima of the $S I$ curves in (b) vs $d_{\text {out }}$ for $C=2,3$, and 4 . Horizontal lines mark the maxima provided by Eq. (3): $C=2$ (red dotted), $C=3$ (green dash dotted), and $C=4$ (blue dashed). Each line intersects the corresponding $S I$ maxima curve at a particular $d_{\text {out }}$, that coincides with the threshold of complete synchronization, as shown by the corresponding vertical lines in (d).

should be close to zero for narrow distributions, while it should take large values for broad distributions reflecting the emergence of complexity. The index $E$ is plotted in Fig. 2(a) (full circles) together with the product of the dynamical indices $S$ and $I$ showing a noticeably similar behavior. It is important to remark that the calculation of $S I$ implies much simpler operations, once the output of system (4) is available.

To more closely inspect the relation between topology and dynamics, we study in Fig. 2(b) the influence of the coupling $d_{\text {out }}$ on the balance between segregation and integration. The main observation is that the number of intercommunity links $\ell_{\text {out }}$ that compromises the balance between the two competing synchronization processes decreases as the link strength $d_{\text {out }}$ increases. We confirm that this trend also holds for modular networks with $C>2$. Namely, we have constructed three and four clique networks and performed the same analysis done for $C=2$, obtaining the fraction $\ell_{\text {out }} / 2 L$ at which the maxima of the $S I$ curves occur. Results are reported in Fig. 2(c) for the three values of $C$ (triangles for $C=4$, squares for $C=3$, and circles for $C=2$ ). Therefore, the functional balance depends on $d_{\text {out }}$ and arises for a given ratio $\ell_{\text {out }} / 2 L$ where the frequency synchronization within the modules is still effective, and at the same time there is significant coordination between the modules characterized by the much richer behavior with the presence of several time scales [as can be observed from Fig. 2(a), where the entropy of the distribution of frequencies exhibits a maximum]. However, the balance between modularity and algebraic connectivity is maximum at one particular value of the mixing ratio as expressed by Eq. (3) [see Fig. 2(c)]. Strikingly, the intersection of the analytical $C$ line $\ell_{\mathrm{out}}^{\max } / 2 L=1-1 / \sqrt{C}$ with the curve of the maxima location of the $S I$ curve predicts the value of $d_{\text {out }}$ that perfectly coincides with the coupling threshold for the onset of the global synchronization, as shown by the vertical lines of Fig. 2(d). Qualitatively similar scenarios have been observed for $3 \mathrm{D}$ chaotic oscillators, and for $2 \mathrm{D}$ excitable units.

In conclusion, we introduced an easily computable topological measure from the knowledge of the adjacency matrix of the graph, and from a given partition, which locates the effective crossover between segregation and integration in a modular network, and shows that its maximum has a dynamical counterpart in the trade-off between the onsets of cluster and complete synchronization of networked phase oscillators. Our results can therefore enlighten the performance of biological systems that have to organize their structure and function to simultaneously perform specialized computations at smaller scales, and bind information at larger ones. As such, it can be applied, in principle, to any real world modular network, namely to evaluate the extent to which a specific configuration has been optimized for information processing, as well as in connection with genetic or simulated annealing algorithms, for the generation of the optimal modular structure with a given number of nodes and links.

This work was supported by the Ministerio de Educación y Ciencia of Spain (FIS2009-07072) and by the Community of Madrid under projects URJC-CM2010-CET-5006 and R\&D Program MODELICO-CM (S2009ESP-1691). The authors acknowledge the computational resources and assistance provided by CRESCO, the center of ENEA in Portici, Italy.

[1] S. Laughlin and T. Sejnowski, Science 301, 1870 (2003).

[2] F. Varela, J. Lachaux, E. Rodriguez, and J. Martinerie, Nat. Rev. Neurosci. 2, 229 (2001).

[3] G. Tononi, O. Sporns, and G. M. Edelman, Proc. Natl. Acad. Sci. U.S.A. 91, 5033 (1994).

[4] P. Fletcher, J. McKenna, K. Friston, C. Frith, and R. Dolan, NeuroImage 9, 337 (1999).

[5] G. Tononi, A.R. McIntosh, D.P. Russell, and G. M. Edelman, NeuroImage 7, 133 (1998).

[6] M. Just, V. Cherkassky, T. A. Keller, and N. Minshew, Brain 127, 1811 (2004).

[7] C. Stam, Clin. Neurophysiol. 116, 2266 (2005).

[8] S. Boccaletti, V. Latora, Y. Moreno, M. Chavez, and D.-U. Hwang, Phys. Rep. 424, 175 (2006). 
[9] A. Arenas, A. Díaz-Guilera, J. Kurths, Y. Moreno, and C. Zhou, Phys. Rep. 469, 93 (2008).

[10] M. Zhao, C. Zhou, Y. Chen, B. Hu, and B.-H. Wang, Phys. Rev. E 82, 046225 (2010).

[11] M. Girvan and M.E. J. Newman, Proc. Natl. Acad. Sci. U.S.A. 99, 7821 (2002).
[12] F. Chung, Spectral Graph Theory, CBMS Regional Conference Series in Mathematics Vol. 92 (American Mathematical Society, Providence, RI, 1997).

[13] M. E. J. Newman and M. Girvan, Phys. Rev. E 69, 026113 (2004).

[14] Y. Kuramoto, Prog. Theor. Phys. 79, 223 (1984). 